\title{
Characterization and evaluation of a recombinant multiepitope peptide antigen MAG in the serological diagnosis of Toxoplasma gondii infection in pigs
}

Yongle Song ${ }^{1,2}$, Yongjuan Zhao ${ }^{1,2}$, Ke Pan $^{1,2}$, Bang Shen ${ }^{1,2}$, Rui Fang ${ }^{2}$, Min Hu, ${ }^{1,2}$ Junlong Zhao ${ }^{1,2}$ and Yanqin Zhou ${ }^{1,2^{*}}$

\begin{abstract}
Background: Toxoplasmosis caused by Toxoplasma gondii is a serious disease threatening human and animal health. People can be infected with T. gondii by ingesting raw pork contaminated with cysts or oocysts. Serological test is a sensitive and specific method usually used for large-scale diagnosis of T. gondii infection in humans and animals (such as pigs). Commercial pig Toxoplasma antibody ELISA diagnostic kits are expensive, which limits their use; moreover, the wide antigen composition used in these diagnostic kits is still unclear and difficult to standardize. The multiepitope peptide antigen is a novel diagnostic marker, and it has potential to be developed into more accurate and inexpensive diagnostic kits.

Methods: The synthetic multiepitope antigen (MAG) CDNA encoding a protein with epitopes from five T. gondiidominant antigens (SAG1, GRA1, ROP2, GRA4, and MIC3) was designed, synthesized, and expressed in Escherichia coli BL21 (DE3) strain. The recombinant protein was detected through western blot with pig anti-T. gondii-positive and -negative serum, and then IgG enzyme-linked immunosorbent assay (ELISA) named MAG-ELISA was designed. The MAG-ELISA was evaluated in terms of specificity, sensitivity, and stability. The MAG-ELISA was also compared with a commercial PrioCHECK ${ }^{\circledR}$ Toxoplasma Ab porcine ELISA (PrioCHECK ELISA). Finally, the trend of pig anti-T. gondii lgG levels after artificial infection with RH tachyzoites was evaluated using MAG-ELISA and two other ELISA methods (rMIC3-ELISA and PrioCHECK ELISA).
\end{abstract}

Results: MAG antigen could be specifically recognized by pig anti-T. gondii-positive but not -negative serum. MAGELISA showed high diagnostic performance in terms of specificity (88.6\%) and sensitivity (79.1\%). MAG-ELISA could be used for detecting anti-T. gondii IgG in the early stage of T. gondii infection in pigs (at least 7 days after artificial infection).

Conclusions: Our results suggest that MAG antigen can be applied to specifically recognize anti-T. gondii IgG in pig, and MAG-ELISA has the potential for large-scale screening tests of T. gondii infection in pig farms and intensive industries.

\footnotetext{
*Correspondence: yanqinzhou@mail.hzau.edu.cn

${ }^{1}$ Key Laboratory Preventive Veterinary of Hubei Province, College

of Veterinary Medicine, Huazhong Agricultural University, Wuhan 430070,

Hubei, People's Republic of China

Full list of author information is available at the end of the article
}

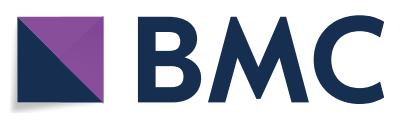

(c) The Author(s) 2021. Open Access This article is licensed under a Creative Commons Attribution 4.0 International License, which permits use, sharing, adaptation, distribution and reproduction in any medium or format, as long as you give appropriate credit to the original author(s) and the source, provide a link to the Creative Commons licence, and indicate if changes were made. The images or other third party material in this article are included in the article's Creative Commons licence, unless indicated otherwise in a credit line to the material. If material is not included in the article's Creative Commons licence and your intended use is not permitted by statutory regulation or exceeds the permitted use, you will need to obtain permission directly from the copyright holder. To view a copy of this licence, visit http://creativecommons.org/licenses/by/4.0/. The Creative Commons Public Domain Dedication waiver (http://creativeco mmons.org/publicdomain/zero/1.0/) applies to the data made available in this article, unless otherwise stated in a credit line to the data. 
Keywords: Toxoplasma gondii, Pig, Synthetic multiepitope antigen, Indirect ELISA, Serological detection

\section{Background}

Toxoplasma gondii is an apicomplexan intracellular protozoan parasite, and it can infect all warm-blooded vertebrates, including humans and domestic animals [1]. This parasite threatens human and animal health especially for pregnant and in immunocompromised individuals [2, 3]. Humans can be infected with $T$. gondii by ingesting food and raw pork contaminated with cysts or oocysts $[4,5]$. Pork is the main meat source in many countries, such as China. Many epidemiological investigations have shown that pig farms and intensive industries have high prevalence and parasite load by PCR detection and serological test, but the detection of T. gondii in pigs is usually not taken seriously in pig farms and intensive industries because of the expense of diagnosis and high error rate [6-8]. Therefore, the development of simple, inexpensive, and sensitive diagnostic tests for $T$. gondii detection in pigs is crucial to reduce the risk of toxoplasmosis in humans and pigs.

The diagnostic approach to toxoplasmosis has been constantly evolving, including traditional techniques (e.g., immunology and imaging tolls) and many emerging molecular techniques. The etiological diagnosis of toxoplasmosis is relatively time-consuming since it involves the isolation of numerous disease materials and requires considerable skills to obtain reliable results. Thus, it is impossible to apply etiological diagnosis to large-scale clinical tests in pig farms and intensive industries. Imaging diagnosis is mainly applied to cerebral and ocular toxoplasmosis using large medical equipment, including computed tomography (CT), magnetic resonance imaging (MRI), nuclear imaging, and ultrasonography (US), but imaging diagnostic results may not be reliable and require expert interpretation [9]. Molecular techniques are widely applied to the epidemiological survey and clinical diagnosis of toxoplasmosis because of their accuracy and sensitivity [10]. The molecular technique used for toxoplasmosis diagnosis is a high-sensitivity nucleic acid detection method for parasites in biological samples, and it overcomes the limitations of the serological tests; in addition, molecular techniques mainly include PCR, nested PCR, real-time PCR, loop-mediated isothermal amplification (LAMP), and recombinase polymerase amplification (RPA) assay [11-13]. However, parasite nucleic acid detection involving DNA extraction tends to be expensive, and it is only accessible in the laboratory. Immunological detection is common method to determine the immune status of the host by examining the change patterns of several different specific antibodies
(IgA, IgM, IgG and IgE) after T. gondii infection [1, 14]. The common immunological method of toxoplasmosis diagnosis includes enzyme-linked immunosorbent assays (ELISA), modified agglutination test (MAT), and others [15-17].

ELISA is a serological detection that can be easily performed on a large scale, and many commercial kits are available to detect specific immunoglobulins (Igs) after T. gondii infection. The solid-phase antigen used for ELISA includes crude tachyzoite antigen, Escherichia coli recombinant antigen, and chimeric peptide antigen. Although Toxoplasma lysate antigen (TLA) has high sensitivity and specificity levels in ELISA, there are problems with TLA such as false-positive results, standardization difficulty, unclear antigen composition, and complex and expensive TLA preparation $[18,19]$. It is impossible to detect all serologically positive individuals by using one or several Escherichia coli recombinant antigens, because the expression patterns of antigen genes from different $T$. gondii strains vary during different infection stages [20]. Synthetic multiepitope antigen, also known as chimeric antigen, is a new generation of recombinant product for ELISA, and it contains multiple immunoreactive epitopes from several dominant antigens of $T$. gondii. Multiepitope antigens have been widely used for toxoplasmosis diagnosis; for example, synthetic multiepitope antigens are applied to detect anti- $T$. gondii $\operatorname{IgG}$ and $\operatorname{IgM}$, and AMA1-SAG2-GRA1-ROP1 chimeric antigens are used to detect specific antibodies of human and mouse in early and chronic T. gondii infection [21]. Chimeric antigen technology has been developed for the serological diagnosis of Trypanosoma cruzi infection caused by another protozoan parasite, cutaneous anthrax caused by Bacillus anthracis, and others [22-24]. However, few studies have been conducted to evaluate chimeric antigens for serodiagnosis of $T$. gondii in pigs and to design an ELISA kit using synthetic antigens for the large-scale diagnosis of toxoplasmosis in pig farms and intensive industries.

Many T. gondii proteins are mainly secreted outside through three specific organelles (rhoptry, dense granule, and microneme), some of which could well activate the host immune system. T. gondii surface antigen 1 (SAG1), as a highly immunogenic protein, is mainly distributed on the tachyzoite surface by glycosyl-phosphatidylinositol anchoring [25, 26]. Dense granule proteins 1 and 4 (GRA1 and GRA4) secreted by $T$. gondii have good antigenicity [27-30]. Rhoptry protein 2 (ROP2) belonging to ROP2-protein family is expressed in three stages (tachyzoites, bradyzoites, and 
sporozoites) of $T$. gondii's life cycle, and this protein induces a strong antibody response in mice and humans [31, 32]. Microneme protein 3 (MIC3), as an adhesion molecule expressed in T. gondii, could be recognized by anti- $T$. gondii-positive serum. Mice immunized with recombinant pseudorabies viruses expressing MIC3 can produce high levels of anti-T. gondii IgG to provide effective protection against $T$. gondii challenge in $\mathrm{BALB} / \mathrm{c}$ mouse model [33]. Although these antigens (SAG1, GRA1, ROP2, GRA4, and MIC3) have been well documented to stimulate host immunity, little work has been done to determine whether the chimeric antigen with their $\mathrm{T}$ and/or B cell epitopes is a good diagnostic marker for toxoplasmosis in pig farms and intensive industries.

To develop an efficient and inexpensive ELISA kit for toxoplasmosis diagnosis in pigs, our study tried to synthesize a multiepitope antigen (MAG) cDNA from five T. gondii-dominant antigens (SAG1, GRA1, ROP2, GRA4 and MIC3) and evaluate the possibility of applying the chimeric protein MAG to the ELISA diagnosis of T. gondii infection in pigs.

\section{Methods}

\section{MAG recombinant chimeric antigen}

The epitope was selected according to two parts. The T/B-cell epitope of each antigen was predicted by Immune Epitope Database (IEDB) with default parameters, and the antigen was also analyzed by DNAstar Lasergene 11 with default parameters. MAG was designed by concatenating peptides containing $\mathrm{T}$ and/ or B cell epitopes (Table 1, Fig. 1a). The MAG cDNA used in the indirect ELISA was synthesized (TSINGKE Biological Technology), and then the synthetic cDNA fragment was cloned into the pGEX-KG expression vector and expressed in Escherichia coli BL21(DE3) strain. MAG recombinant protein fused with glutathione S-transferase (GST) was induced with IPTG (isopropyl $\beta$-D-1-thiogalactopyranoside) and then purified by glutathione-based affinity chromatography (GE Healthcare

Table 1 Sources of chimeric antigen MAG

\begin{tabular}{lllll}
\hline Protein & Epitope & Strain & Sequence & Position \\
\hline SAG1 & S1 & GT1 & TCPDKKSTA & 59-67 \\
& S2 & GT1 & ILPKLTENPWQ & $246-256$ \\
GRA1 & G1 & GT1 & DTMKSMQRDED & $104-114$ \\
ROP2 & R2 & GT1 & PGDVIEELFNRIPETSV & $197-214$ \\
GRA4 & G4 & GT1 & SGLTGVKDSSS & $235-245$ \\
MIC3 & M3 & GT1 & KRTGCHAFRE...SCKCDNGYSG & 233-310 \\
\hline
\end{tabular}

Life Sciences, USA). Finally, the purified MAG antigens were quantified by enhanced bicinchoninic acid (BCA) protein assay kit (Beyotime Biotechnology, China).

\section{Western immunoblot analysis}

The purified MAG protein was separated by SDS-PAGE to identify the protein molecular weight through protein molecular weight marker (ThermoFisher scientific) and analyzed by western blot. The Immobilon-PSQ PVDF membrane $(0.2 \mu \mathrm{m}$ pore size, Millipore, USA) onto which purified protein was transferred was incubated with pig anti-T. gondii-positive or -negative serum and then detected with horseradish peroxidase (HRP)-conjugated rabbit anti-swine IgG $(\mathrm{H}+\mathrm{L})$ (Frdbio Bioscience \& Technology, China).

\section{Pig artificial infection with tachyzoite}

"Chang xin" binary miscellaneous commodity pigs (any gender, weight of $15-20 \mathrm{~kg}$ ) bred by the pig farm of Huazhong Agricultural University were used to perform RH (type I strain) tachyzoite artificial infection by intraperitoneal injection. Four pigs were randomly assigned into two groups with two pigs in the infected group and two in the control group. Two pigs in the infected group were intraperitoneally injected with 5 million tachyzoites for T. gondii infection. The two pigs in the control group were intraperitoneally injected with an equal volume of sterile $0.9 \% \mathrm{NaCl} ; 2 \mathrm{ml}$ of serum samples was collected from the infected and control group on days $0,2,4,7,14$, $21,28,35,42$, and 49 . The serum sample at day 3 before T. gondii infection was also collected for subsequent detection.

\section{Optimization of ELISA procedure}

ELISA was performed as described previously [20]. The 96-well flat-bottom microtiter plates (BIOFIL, China) were coated overnight at $4{ }^{\circ} \mathrm{C}$ with $100 \mu \mathrm{l}$ per well of coating buffer ( $25 \mathrm{mM}$ carbonate buffer, $\mathrm{pH}$ 9.6) containing MAG-purified antigen. The 96-well plate was washed three times with wash buffer (phosphate-buffered saline (PBS) with $0.05 \%$ Tween 20). Blocking buffer (bovine serum albumin (BSA) in PBS) was added, and then the plate was incubated for a period of time at $37^{\circ} \mathrm{C}$. After three rewashes as described above, $100 \mu \mathrm{l}$ of pig serum diluted with serum dilution buffer was added to each well, and the plates were incubated for a period of time at $37^{\circ} \mathrm{C}$. After being washed as described above, $100 \mu \mathrm{l}$ of HRP conjugated recombinant Protein A/G (ThermoFisher Scientific) diluted in blocking buffer was added to each well, and the plates were incubated for a period of time at $37^{\circ} \mathrm{C}$. After being washed as described above, color was developed for a period of time at $37^{\circ} \mathrm{C}$ in a dark room after the addition of $100 \mu \mathrm{l}$ per well of the substrate 


\section{a}
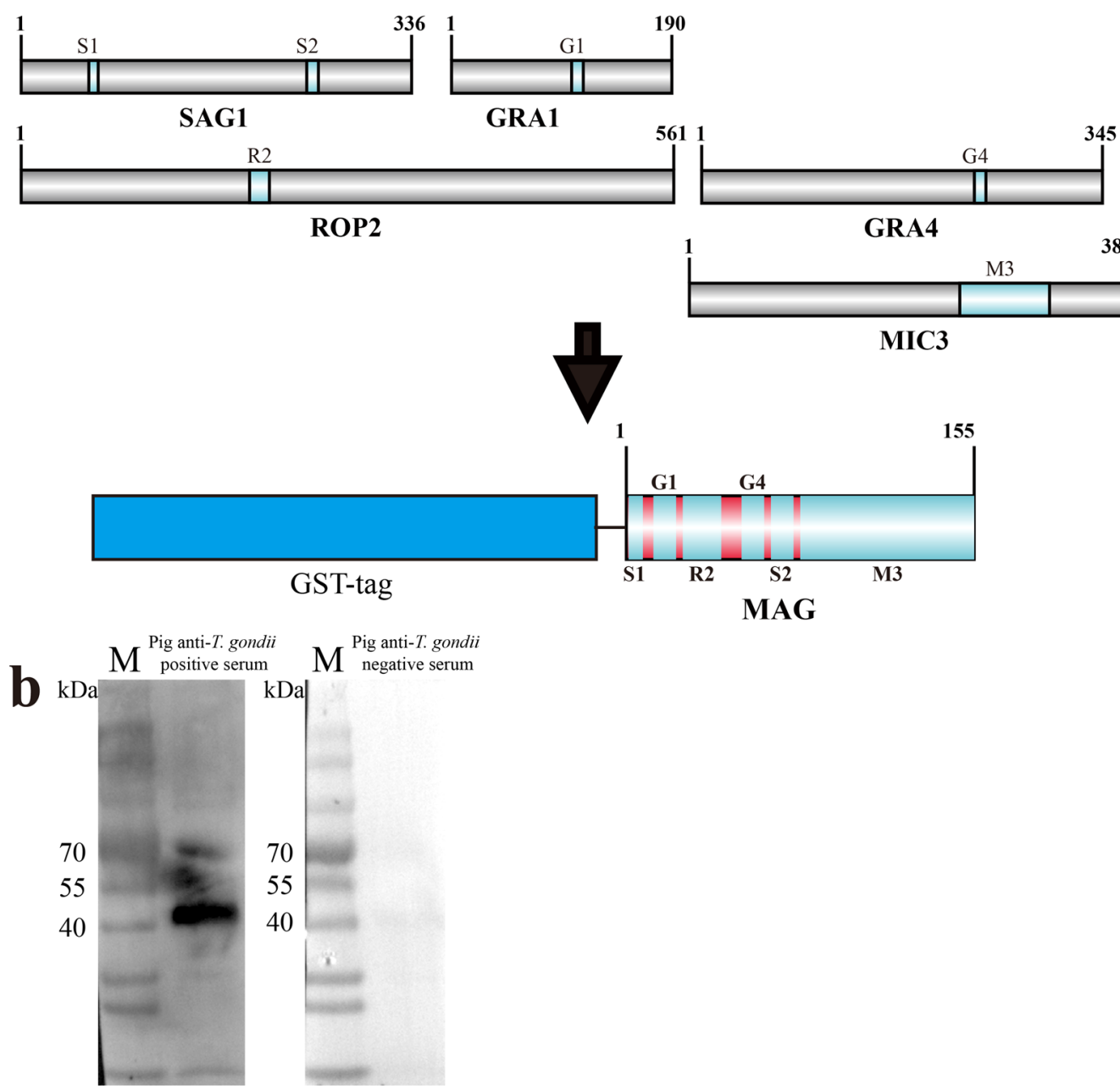

Fig. 1 MAG construction and antigen identification. a Construction of chimeric antigen MAG. b Western blot to identify MAG antigenicity

solution containing 3,3',5,5'-tetramethylbenzidine (TMB, Sigma-Aldrich, USA) and $\mathrm{H}_{2} \mathrm{O}_{2}$. Finally, the OD630 value was measured with a Bio-Tek ELx-800 microplate reader (BioTek Instruments, USA). All the tests were performed in duplicate wells.

To obtain the optimal dilution ratio of MAG-coated antigen and pig serum, the cross-titration was performed at serial dilution ratios of MAG antigen (1:100, 1:200, 1:400, 1:800, 1:1600, and 1:3200), T. gondii-positive serum, and negative pig serum samples $(1: 20,1: 40,1: 80$, $1: 160,1: 320$, and 1:640). The OD630 was measured, and the ratio of positive/negative serum $(\mathrm{P} / \mathrm{N})$ was calculated at different dilution ratios. The dilution ratios of MAG antigen and pig serum with the maximum value of $\mathrm{P} / \mathrm{N}$ were designated to be the optimal dilution ratio.

The optimal blocking buffer concentration $(0.5 \%, 1.0 \%, 1.5 \%$, and $2.0 \% \mathrm{BSA})$ and the optimal blocking time $(30,40,50$, and $60 \mathrm{~min})$ were determined as described above. The optimal serum dilution buffer $(0.1 \%$ BSA, 0.5\% BSA, and PBS containing 0.05\% Tween 20) was determined by the maximum value of $\mathrm{P} / \mathrm{N}$. The optimal coating time of pig serum $(30,40,50$, and $60 \mathrm{~min}$ ) was determined by the maximum value of $\mathrm{P} / \mathrm{N}$ under the above optimal conditions. The optimal dilution ratio $(1: 3000,1: 4000,1: 5000,1: 6000,1: 7000$ and 1:8000) and the optimal reaction time (30, 40, 50 and $60 \mathrm{~min}$ ) of HRP-conjugated recombinant Protein A/G were also determined by cross-titration. The optimal reaction time of TMB substrate solution $(5,7.5,10$, and $12.5 \mathrm{~min}$ ) was determined by the maximum value of $\mathrm{P} / \mathrm{N}$. The cutoff value of MAG-ELISA was determined referring to the OD630 mean value of $25 \mathrm{~T}$. gondii-negative pig sera under optimal conditions (cutoff $=$ mean $+3 \mathrm{SD})$. 


\section{Evaluation of MAG-ELISA}

The cross-reactivity of MAG-ELISA was assessed by the diagnosis of five common pig virus sera [swine fever virus (SFV), porcine reproductive and respiratory disease virus (PRRSV), pseudorabies virus (PrV), porcine circovirus (PCV), and foot-and-mouth disease virus (FMDV)] and the control group including pig anti- $T$. gondii-positive and -negative sera. The lowest detection limit of MAGELISA was determined by measuring OD630 at serial dilution concentrations of pig anti-T. gondii-positive sera. The coefficient of variation $(\mathrm{CV})$ of repeated tests within batches and between batches was calculated and confirmed respectively using six samples (one anti- $T$. gondiipositive and five -negative pig sera). The stability was tested by the destruction experiments in which MAGcoated plates were placed at $37^{\circ} \mathrm{C}$ for $12,24,36,48,60$, and $72 \mathrm{~h}$, respectively. The 209 pig serum samples were tested by MAG-ELISA and a commercial PrioCHECK ${ }^{\circledR}$ Toxoplasma Ab porcine ELISA (Prionics, Switzerland), respectively. The OD450 cutoff value of PrioCHECK ELISA was calculated according to the manual. The sensitivity and specificity of MAG-ELISA versus PrioCHECK ELISA were assessed according to two ELISA results.

\section{Procedure of rMIC3-ELISA}

The rMIC3-ELISA was performed in a previously reported ELISA method for detecting pig toxoplasmosis $[34,35]$. Entire recombinant T. gondii microneme protein 3 (rMIC3) fused with GST tag was expressed and purified as coated antigen. The 96-well plates were coated with rMIC3 at $3.40 \mu \mathrm{g} / \mathrm{ml}$, and pig serum samples were diluted at 1:160. The cutoff value of rMIC3-ELISA was defined as 0.40 . The OD630 value was measured as described above.

\section{Results}

Characterization of recombinant multiepitope antigen and optimization of MAG-ELISA procedure

Our study selected six epitopes from five reported $T$. gondii-dominant antigens (SAG1, GRA1, ROP2, GRA4, and MIC3) and designed a new chimeric recombinant multiepitope antigen (MAG) by concatenating peptides containing $\mathrm{T}$ and/or B cell epitopes (Table 1, Fig. 1a). MAG was synthesized and cloned into pGEX-KG vector for prokaryotic expression. The MAG protein with GST tag (43.3KD) was successfully expressed in Escherichia coli BL21 (DE3) and purified through glutathione-based affinity chromatography. Western blot results showed the MAG protein was recognized specifically by pig anti- $T$. gondii-positive serum but not by -negative serum (Fig. 1b).

To improve the performance of MAG-ELISA, we attempted to optimize the procedures related to MAG antigen, pig serum, buffer, secondary antibody, and
ELISA substrate, and others. The cross-titration showed that the optimal dilution ratio of MAG-coated antigen was 1:100 $(0.91 \mu \mathrm{g} /$ well $)$ and that of pig serum was 1:160 (Fig. 2a). The maximum $\mathrm{P} / \mathrm{N}$ was obtained when a MAG-coated plate was incubated with $1.0 \%$ BSA for 30 min under the optimal conditions as described above (Fig. 2b). We also found 0.5\% BSA was the optimal serum dilution buffer compared to $0.1 \%$ BSA and PBS containing $0.05 \%$ Tween 20 (Fig. 2c). The optimal pig serum incubation time was determined to be $40 \mathrm{~min}$ (Fig. 2d). HRP-conjugated recombinant Protein A/G exhibited optimal performance when the plates were incubated at 1:8000 for $60 \mathrm{~min}$ (Fig. 2e). The optimal color development was obtained after TMB substrate was incubated for $10 \mathrm{~min}$ (Fig. 2f). The cutoff value of MAG-ELISA was set as 0.25 after testing $25 \mathrm{~T}$. gondii negative pig sera under the optimal conditions described above (Fig. 2g). The subsequent MAG-ELISA was performed according to the above-mentioned optimal procedures.

\section{Evaluation of MAG-ELISA}

To evaluate the feasibility of our MAG-ELISA, we also examined its specificity, sensitivity, stability, and others. The OD630 values of the five common pig viruses (SFV, PRRSV, PrV, PCV, and FMDV) were significantly lower than the cutoff value of MAG-ELISA (Fig. 3a), indicating our ELISA did not exhibit obvious cross-reaction with these five common pig viruses, which confirmed the good specificity of MAG-ELISA. By measuring OD630 at serial dilutions of T. gondii-positive pig serum, we found that pig anti-T. gondii-positive serum was still determined as positive even at the dilution ratio of 1:320, indicating the sensitivity of MAG-ELISA was adequate (Fig. $3 \mathrm{~b}$ ). The $\mathrm{CV}$ of repeated tests within batches and between batches was all $<10.0 \%$, suggesting the good stability of our MAG-ELISA (Fig. 3c). After placing MAGcoated plates at $37^{\circ} \mathrm{C}$ for $72 \mathrm{~h}$, the OD630 only dropped by $<20.0 \%$ (Fig. 3d). We further compared MAG-ELISA with PrioCHECK ELISA by testing 209 pig serum samples simultaneously. The sensitivity of MAG-ELISA was $79.1 \%$, specificity was $88.6 \%$, positive predictive value (PPV) was $82.9 \%$, and negative predictive value (NPV) was $85.8 \%$ (Table 2, Additional file 1: Tables S1 and S2).

\section{MAG-ELISA detection of artificial pig infection with type I tachyzoite}

MAG-ELISA and two other ELISA methods (rMIC3ELISA and PrioCHECK ELISA) were applied to detect pig anti-T. gondii IgG level after artificial infection with $\mathrm{RH}$ tachyzoites. We collected pig serum samples from infected group and control group at days $0,2,4,7,14,21,28,35,42$, and 49 after infection and at day 3 before infection, respectively. MAG-ELISA 


\section{$\mathbf{a}$}

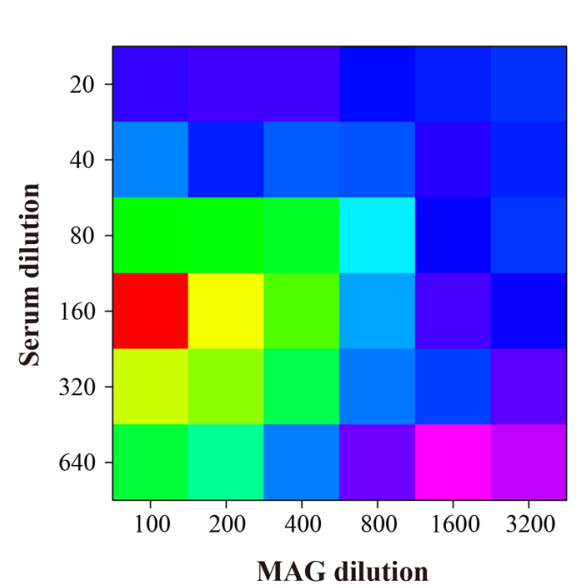

C

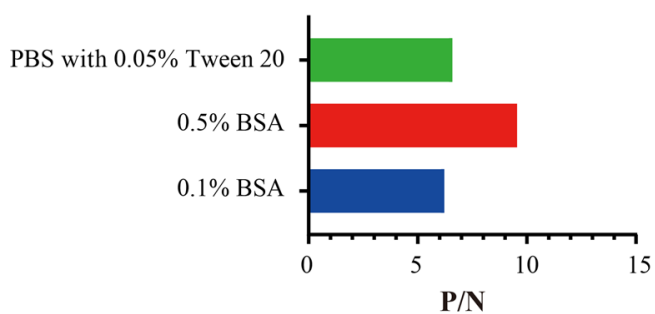

$\mathbf{e}$

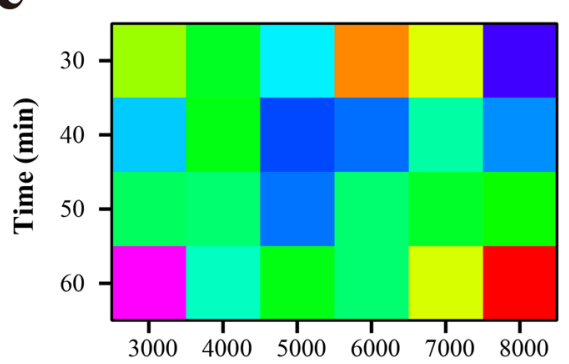

HRP conjugated recombinant protein $\mathrm{A} / \mathrm{G}$ dilution

$\mathbf{P} / \mathbf{N}$

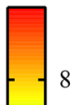

6

10.5 b

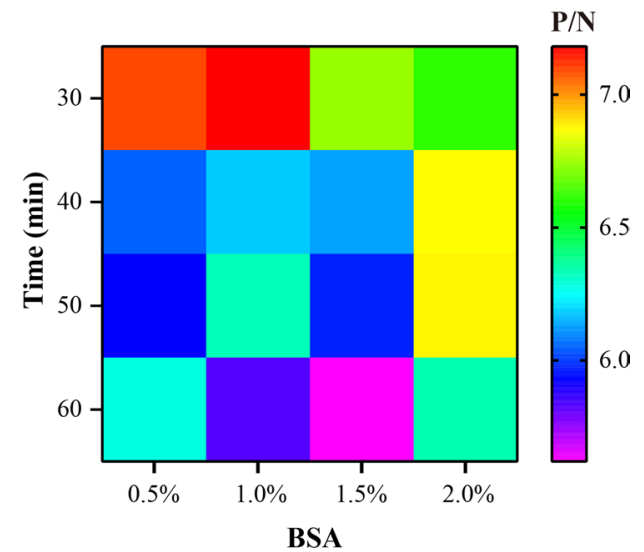

d
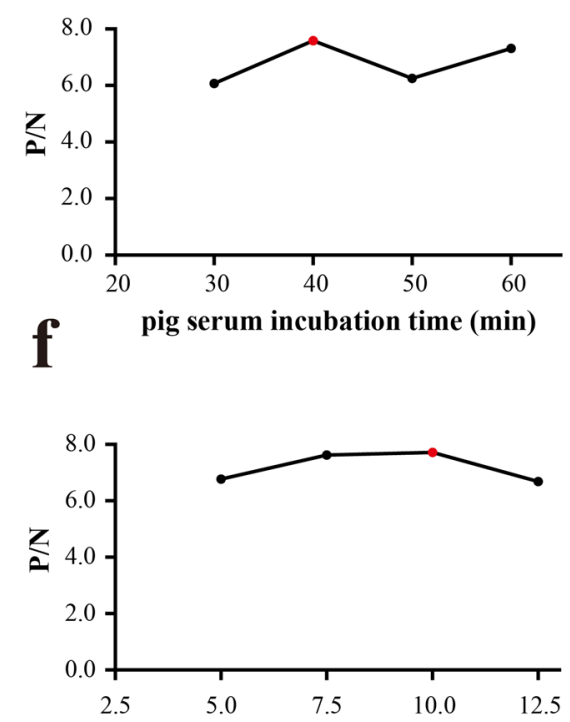

TMB substrate incubation time (min)

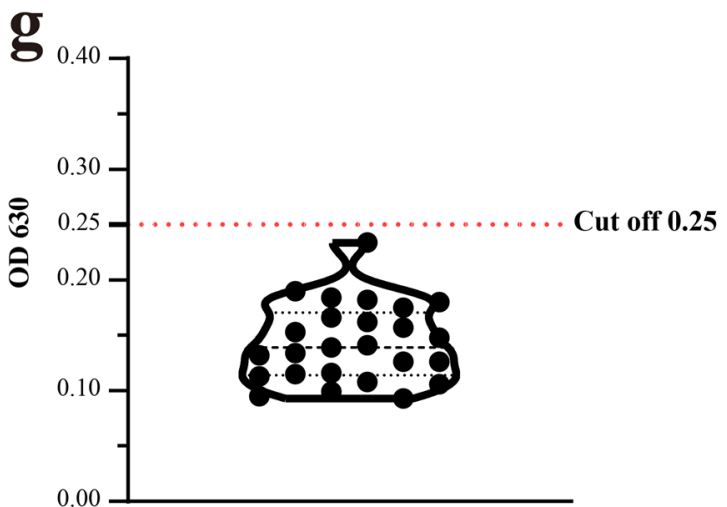

Fig. 2 Optimization of MAG-ELISA. a Optimal dilution ratio of MAG antigen and pig serum. b Blocking buffer optimization of BSA concentration and incubation time. $\mathbf{c}$ Optimization of pig serum dilution buffer. $\mathbf{d}$ Optimization of pig serum incubation time. e Optimization of HRP conjugated recombinant Protein A/G dilution ratio and incubation time. $\mathbf{f}$ Optimization of TMB substrate incubation time. $\mathbf{g}$ Cutoff value of pig anti-Toxoplasma gondii-negative serum (cutoff $=$ mean $+3 \mathrm{SD})$ 


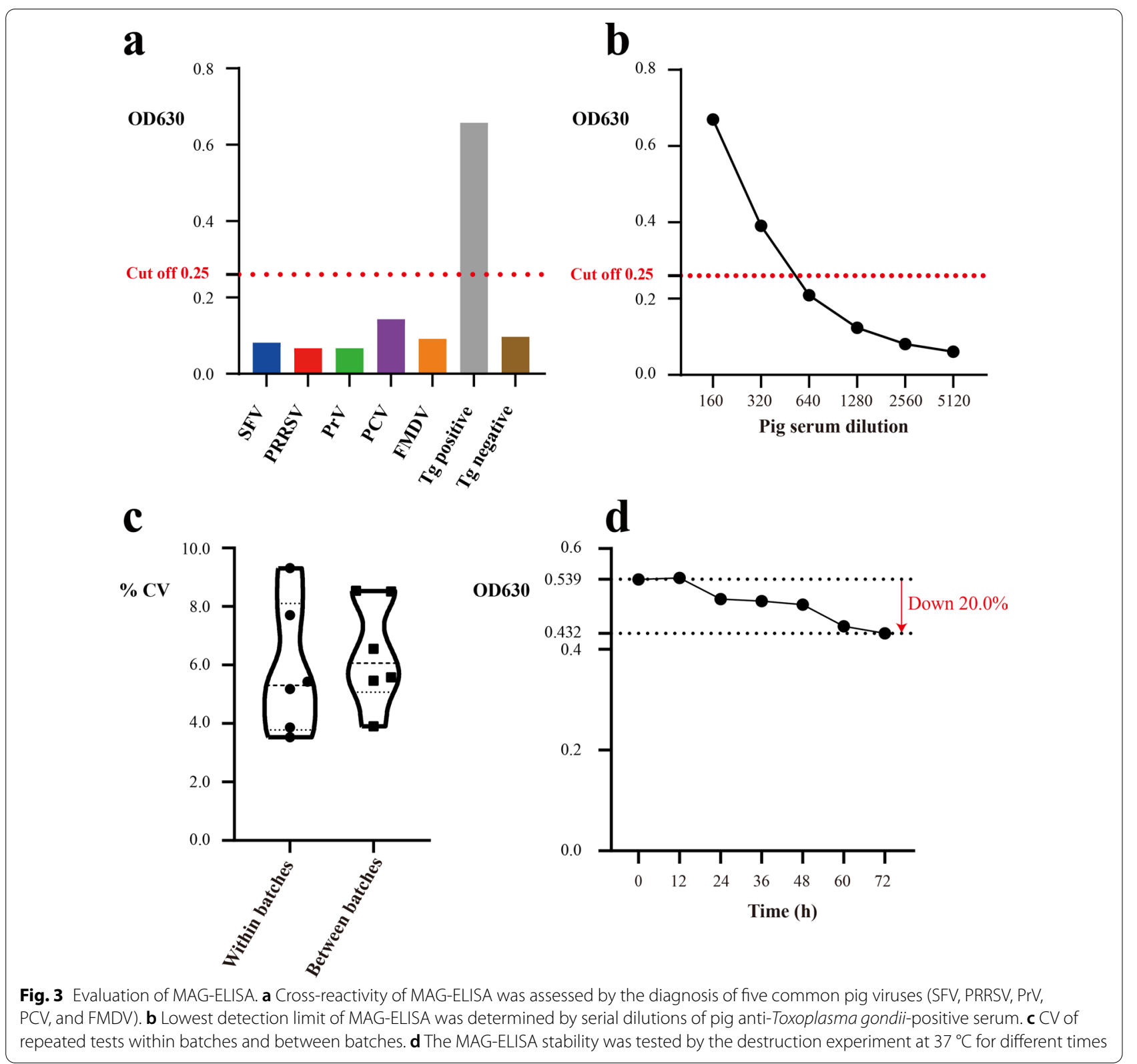

Table 2 Comparison of MAG-ELISA and PrioCHECK ELISA to detect Toxoplasma gondii infection

\begin{tabular}{lllr}
\hline & \multicolumn{2}{l}{ PrioCHECK ELISA } & \\
\cline { 2 - 3 } & Positive & Notal \\
\hline MAG-ELISA & & 14 & \\
Positive & 68 & 109 & 82 \\
Negative & 18 & 123 & 127 \\
Total & 86 & & 209 \\
\hline
\end{tabular}

detected anti-T. gondii-positive IgG in the early stage of pig infection (at least 7 days), which was earlier than the results obtained by rMIC3-ELISA and PrioCHECK ELISA at days 35 and 14, respectively (Fig. 4). Two weeks after artificial infection, our MAG-ELISA indicated that the overall level of anti-T. gondii IgG had gradually decreased, but rMIC3-ELISA and PrioCHECK ELISA showed that the level of anti-T. gondii IgG reached its maximum value after day 35 postinfection. Therefore, MAG-ELISA could be applied for early detection of $T$. gondii infection in pig farms and intensive industries. 


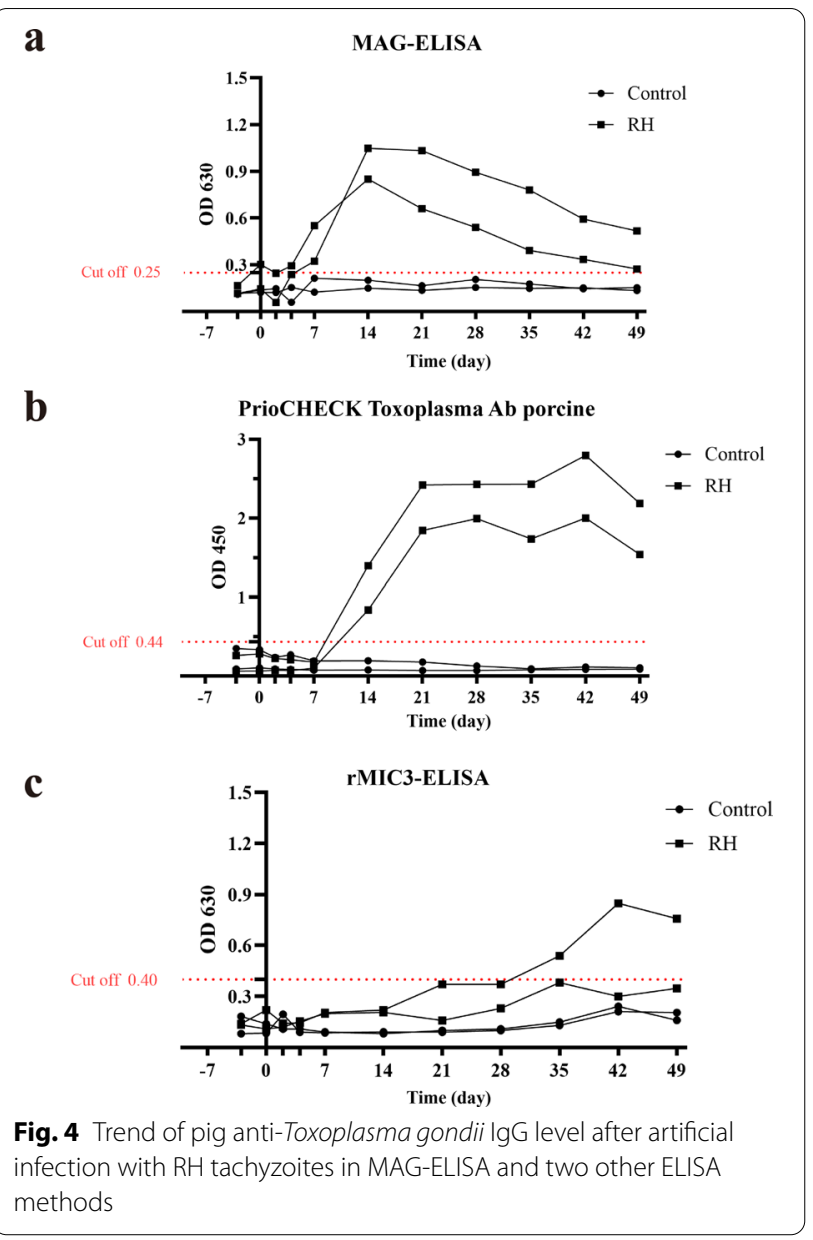

\section{Discussion}

Humans can be infected by T. gondii by digesting raw or uncooked meats (such as pork). Infection with T. gondii can lead to reproductive disorders in sows (such as miscarriage, stillbirths, and weak fetuses). Poor meat quality threatens human health and causes serious economic losses to the livestock industry. T. gondii can survive for a long time in many pig tissues such as heart, lungs, and brain in the form of cysts. Pork is considered to be one of the main sources of human toxoplasmosis [4, 36]. A high prevalence of $T$. gondii is found in pig farms, especially organic farms and intensive pig industries [37, 38]. Therefore, it is necessary to develop a highly sensitive, specific, and inexpensive kit for the large-scale diagnosis of pig toxoplasmosis in farms and intensive industries.

ELISA is a common method for the large-scale detection of infectious disease mainly caused by pathogenic bacteria, viruses, and parasites. The specificity and sensitivity of ELISA depend mainly on the coated antigen. All the common coated antigens such as TLA and recombinant antigen have their own limitations. For example, the composition of TLA is poorly understood, and TLA preparation procedure is complex and can be infectious for the operator. Although recombinant protein antigen of $T$. gondii is very useful for serodiagnosis of toxoplasmosis, one recombinant antigen can detect only one type of anti-T. gondii IgG, and the diagnosis result may be affected by the expression level of antigen gene [39]. Thus, the application of these antigens in ELISA may cause a high error rate in large-scale detection. The chimeric antigen encoded by synthetic multiepitope antigen cDNA is a new generation of recombinant products, and it has competitive advantages since it contains more immunoreactive epitopes from several dominant antigens of $T$. gondii than conventional recombinant antigen. Chimeric antigen with the epitope from no more than three T. gondii antigens used in ELISA has been reported to perform well in toxoplasmosis diagnosis [19]. To further improve the performance of ELISA, our MAG-ELISA used a chimeric antigen containing six epitopes of $T$ and $B$ cells from five T. gondii-dominant antigens (SAG1, GRA1, ROP2, GRA4, and MIC3). SAG1 is only highly expressed in acute infection, but GRA1, ROP2, ROP4, and MIC3 are expressed in acute and chronic T. gondii infection, endowing MAG antigen with the potential to detect pig anti-T. gondii IgG in two different infection periods. Our results showed that MAG-ELISA possessed high specificity, sensitivity, and repeatability. The test of 209 pig serum samples indicated that the sensitivity of MAG-ELISA was $79.1 \%$, specificity was $88.6 \%$, PPV was $82.9 \%$, and NPV was $85.8 \%$ (Table 2, Additional file 1: Tables S1 and S2). The seroprevalence of $T$. gondii infection between MAGELISA (39.2\%) and PrioCHECK ELISA (41.1\%) showed little difference, indicating that MAG-ELISA is valuable for large-scale screening of $T$. gondii infection in pig farms. The diversity of test results between MAG-ELISA and PrioCHECK ELISA was mainly related to the weak positive and negative samples in PrioCHECK ELISA. The accuracy of serological testing methods is always limited by the level of antibodies detected in the serum samples. The antigen used in commercial PrioCHECK ELISA was formalin-fixed cell culture-derived tachyzoite antigen, but the antigen used in MAG-ELISA was several peptides from five T. gondii-dominant antigens (SAG1, GRA1, ROP2, GRA4, and MIC3). The more types of antigens in commercial kits, the greater the potential for the detection of T. gondii infection in pigs, but for PrioCHECK ELISA antigens with unclear components, false positives are inevitable. The low total IgG level in MAG-ELISA might be attributed to the inconsistence in the levels of anti- T. gondii individual IgGs against these six epitopes at different infection stages. Furthermore, false-positive results of the PrioCHECK ELISA also might be caused by some sample test results being close to the cutoff value because of the unclear component of TLA antigen. It is 
possible to improve the sensitivity and specificity of the recombinant multiepitope peptide antigen in the serological diagnosis of $T$. gondii infection by using a large number of peptides that are highly expressed during acute and chronic infection.

We further compared the obtained levels of anti-T. gondii IgGs after artificial infection with RH tachyzoites through three different ELISAs (MAG-ELISA, rMIC3ELISA, and PrioCHECK ELISA). Our result showed that MAG-ELISA could detect $T$. gondii infection at day 7 after infection, which was earlier than the other two ELISA methods. However, the level of anti-T. gondii IgG obtained through MAG-ELISA was inconsistent with those through rMIC3-ELISA and PrioCHECK (Fig. 4). The reasons for the above-mentioned inconsistency might be as follows. First, pig anti-T. gondii IgGs against the epitopes of SAG1, GRA1, ROP2, GRA4, and MIC3, respectively, had inconsistent levels after artificial $\mathrm{RH}$ infection. Second, the IgG trend in MAG-ELISA could represent the overall level of several anti-T. gondii IgGs. Thus, our MAG-ELISA should be more reliable than the ELISA using only one recombinant antigen. However, the MAG-ELISA could detect anti- $T$. gondii IgG only within 1 to 7 weeks after artificial infection with a detection time span shorter than that of the other two ELISA methods, which might be due to the low expression of the antigen genes corresponding to some epitopes. Previous study also indicated that antibody responses to GRA1 and MIC3 were very weak or even absent 6 weeks post infection [40].

MAG could specifically recognize pig anti- $T$. gondii IgG; thus, MAG-ELISA has potential for diagnosing Toxoplasma infection in pigs. MAG-ELISA could help improve the accuracy and reduce the cost of toxoplasmosis diagnosis in pig farms and intensive industries. MAG-ELISA can monitor the trend of change in antiT. gondii IgG after artificial pig infection, increasing our knowledge about $T$. gondii infection in pigs. Further work is also needed to optimize the performance of the MAGELISA and verify its diagnostic effect in detecting acute and chronic T. gondii infection in pigs and other animals (such as pets).

\section{Conclusions}

We report that MAG, as a synthetic multiepitope antigen, can recognize pig anti- $T$. gondii-positive but not -negative serum. MAG-ELISA had good diagnostic performance in terms of specificity and sensitivity compared with a commercial PrioCHECK ELISA. MAG-ELISA has potential for use in large-scale diagnosis of $T$. gondii infection in pig farms and intensive industries.

\section{Abbreviations}

MAG: Synthetic multiepitope antigen; ELISA: Enzyme-linked immunosorbent assay; PrioCHECK ELISA: PrioCHECK ${ }^{\circledR}$ Toxoplasma Ab porcine ELISA; TLA: Toxoplasma lysate antigen; SAG1: T. gondii surface antigen 1; GRA1: Dense granule protein 1; GRA4: Dense granule protein 4; ROP2: Rhoptry protein 2; MIC3: Microneme protein 3; BCA: Bicinchoninic acid; HRP: Horseradish peroxidase; IPTG: Isopropyl $\beta$-D-1-thiogalactopyranoside; GST: Glutathione S-transferase; PBS: Phosphate buffered saline; BSA: Bovine serum albumin; TMB: 3,3',5,5'Tetramethylbenzidine; P/N: Positive/negative serum; SFV: Swine fever virus; PRRSV: Porcine reproductive and respiratory disease virus; PrV: Pseudorabies virus; PCV: Porcine circovirus; FMDV: Foot-and-mouth disease virus; CV: Coefficient of variation; rMIC3: Recombinant entire T. gondii microneme protein 3; PPV: Positive predictive value; NPV: Negative predictive value.

\section{Supplementary Information}

The online version contains supplementary material available at https://doi. org/10.1186/s13071-021-04917-w.

Additional file 1: Table S1. Diagnosis results of 209 pig serum samples by MAG-ELISA. Table S2. Diagnosis results of 209 pig serum samples by PrioCHECK ELISA.

\section{Acknowledgements}

We thank the Laboratory Animal Center of Huazhong Agricultural University for its services. Our gratitude also goes to linguistics professor Ping Liu from Huazhong Agriculture University, Wuhan, China, for her work in English editing and language polishing.

\section{Authors' contributions}

YQZ conceived this project, designed the experiment and reviewed the manuscript. YLS, YJZ and KP performed the experiment, analyzed and interpreted the data and drafted the manuscript. BS, RF, MH and JLZ participated in designing the experiment and reviewing the manuscript. All authors read and approved the final manuscript.

\section{Funding}

Our work was supported by the National Key Research and Development Program of China (grant no. 2017YFD0501304) and the National Natural Science Foundation of China (grant no. 31272553).

Availability of data and materials

All relevant data are within the paper and additional file.

\section{Declarations}

\section{Ethics approval and consent to participate}

Our study was authorized by the animal ethical committee from Huazhong Agricultural University in China with the number HZAUSW-2012-001. Two hundred nine pig serum samples were collected from pig farms in Hubei Province and pigs artificially infected with tachyzoite and stored in the animal parasite laboratory of Huazhong Agricultural University. Pigs for T. gondii artificial infection were provided by the pig farm of Huazhong Agricultural University. All pigs were killed after the T. gondii infection experiment.

\section{Consent for publication}

Not applicable.

\section{Competing interests}

The authors declare that they have no competing interests.

\section{Author details}

${ }^{1}$ Key Laboratory Preventive Veterinary of Hubei Province, College of Veterinary Medicine, Huazhong Agricultural University, Wuhan 430070, Hubei, People's Republic of China. ${ }^{2}$ State Key Laboratory of Agricultural Microbiology, Huazhong Agricultural University, Wuhan 430070, Hubei, People's Republic of China. 
Received: 31 May 2021 Accepted: 2 August 2021

Published online: 17 August 2021

\section{References}

1. Robert-Gangneux F, Dardé ML. Epidemiology of and diagnostic strategies for toxoplasmosis. Clin Microbiol Rev. 2012;25:264-96.

2. Pappas G, Roussos N, Falagas ME. Toxoplasmosis snapshots: global status of Toxoplasma gondii seroprevalence and implications for pregnancy and congenital toxoplasmosis. Int J Parasitol. 2009:39:1385-94.

3. Torgerson PR, Mastroiacovo P. The global burden of congenital toxoplasmosis: a systematic review. Bull World Health Organ. 2013;91:501-8.

4. Gisbert Algaba I, Verhaegen B, Jennes M, Rahman M, Coucke W, Cox E, et al. Pork as a source of transmission of Toxoplasma gondii to humans: a parasite burden study in pig tissues after infection with different strains of Toxoplasma gondii as a function of time and different parasite stages. Int J Parasitol. 2018;48:555-60.

5. Condoleo R, Rinaldi L, Sette S, Mezher Z. Risk assessment of human toxoplasmosis associated with the consumption of pork meat in Italy. Risk Anal. 2018;38:1202-22.

6. Santoro M, Viscardi M, Sgroi G, D'Alessio N, Veneziano V, Pellicano R, et al. Real-time PCR detection of Toxoplasma gondii in tissue samples of wild boars (Sus scrofa) from southern Italy reveals high prevalence and parasite load. Parasit Vectors. 2019;12:335.

7. Foroutan M, Fakhri Y, Riahi SM, Ebrahimpour S, Namroodi S, Taghipour A, et al. The global seroprevalence of Toxoplasma gondii in pigs: a systematic review and meta-analysis. Vet Parasitol. 2019;269:42-52.

8. Tao Q, Wang Z, Feng H, Fang R, Nie H, Hu M, et al. Seroprevalence and risk factors for Toxoplasma gondii infection on pig farms in central China. J Parasitol. 2011:97:262-4.

9. Rostami A, Karanis P, Fallahi S. Advances in serological, imaging techniques and molecular diagnosis of Toxoplasma gondii infection. Infection. 2018;46:303-15

10. Switaj K, Master A, Skrzypczak M, Zaborowski P. Recent trends in molecular diagnostics for Toxoplasma gondii infections. Clin Microbiol Infect. 2005;11:170-6.

11. Gutierrez J, O'Donovan J, Williams E, Proctor A, Brady C, Marques PX, et al. Detection and quantification of Toxoplasma gondii in ovine maternal and foetal tissues from experimentally infected pregnant ewes using real-time PCR. Vet Parasitol. 2010;172:8-15.

12. Fallahi S, Seyyed Tabaei SJ, Pournia Y, Zebardast N, Kazemi B. Comparison of loop-mediated isothermal amplification (LAMP) and nested-PCR assay targeting the RE and B1 gene for detection of Toxoplasma gondii in blood samples of children with leukaemia. Diagn Microbiol Infect Dis. 2014;79:347-54.

13. Wu YD, Xu MJ, Wang QQ, Zhou CX, Wang M, Zhu XQ, et al. Recombinase polymerase amplification (RPA) combined with lateral flow (LF) strip for detection of Toxoplasma gondii in the environment. Vet Parasitol. 2017;243:199-203.

14. Zhang K, Lin G, Han Y, Li J. Serological diagnosis of toxoplasmosis and standardization. Clin Chim Acta. 2016:461:83-9.

15. Turunen $H$, Vuorio KA, Leinikki PO. Determination of IgG, IgM and IgA antibody responses in human toxoplasmosis by enzyme-linked immunosorbent assay (ELISA). Scand J Infect Dis. 1983;15:307-11.

16. Li S, Maine G, Suzuki Y, Araujo FG, Galvan G, Remington JS, et al. Serodiagnosis of recently acquired Toxoplasma gondii infection with a recombinant antigen. J Clin Microbiol. 2000;38:179-84.

17. Desmonts $G$, Remington JS. Direct agglutination test for diagnosis of Toxoplasma infection: method for increasing sensitivity and specificity. J Clin Microbiol. 1980;11:562-8.

18. Pishkari S, Shojaee S, Keshavarz H, Salimi M, Mohebali M. Evaluation of Toxoplasma gondii soluble, whole and excretory/secretary antigens for diagnosis of toxoplasmosis by ELISA test. J Parasit Dis. 2017;41:289-91.

19. Ferra B, Holec-Gąsior L, Kur J. Serodiagnosis of Toxoplasma gondii infection in farm animals (horses, swine, and sheep) by enzyme-linked immunosorbent assay using chimeric antigens. Parasitol Int. 2015;64:288-94.

20. Dai J, Jiang M, Wang Y, Qu L, Gong R, Si J. Evaluation of a recombinant multiepitope peptide for serodiagnosis of Toxoplasma gondii infection. Clin Vaccine Immunol. 2012;19:338-42.

21. Ferra BT, Holec-Gąsior L, Gatkowska J, Dziadek B, Dzitko K, Grąźlewska W, et al. The first study on the usefulness of recombinant tetravalent chimeric proteins containing fragments of SAG2, GRA1, ROP1 and AMA1 antigens in the detection of specific anti-Toxoplasma gondii antibodies in mouse and human sera. PLoS ONE. 2019:14:e0217866.

22. Leony LM, Freitas NEM, Del-Rei RP, Carneiro CM, Reis AB, Jansen AM, et al. Performance of recombinant chimeric proteins in the serological diagnosis of Trypanosoma cruzi infection in dogs. PLoS Negl Trop Dis. 2019;13:e0007545.

23. Varshney A, Puranik N, Kumar M, Pal V, Padmaja J, Goel AK. An ELISA using a recombinant chimera of protective antigen and lethal factor for serodiagnosis of cutaneous anthrax in India. Biologicals. 2019;57:55-60.

24. Cardinal MV, Sartor PA, Gaspe MS, Enriquez GF, Colaianni I, Gürtler RE. High levels of human infection with Trypanosoma cruzi associated with the domestic density of infected vectors and hosts in a rural area of northeastern Argentina. Parasit Vectors. 2018;11:492.

25. Tomavo S, Schwarz RT, Dubremetz JF. Evidence for glycosyl-phosphatidylinositol anchoring of Toxoplasma gondii major surface antigens. Mol Cell Biol. 1989;9:4576-80.

26. Naghi Vishteh M, Javadi Mamaghani A, Rashidi S, Seyyed Tabaei SJ, JeddiTehrani M. Peptide-based monoclonal antibody production against SAG1 (P30) protein of Toxoplasma gondii. Monoclon Antib Immunodiagn Immunother. 2020:39:51-6.

27. Cesbron-Delauw MF, Guy B, Torpier G, Pierce RJ, Lenzen G, Cesbron JY, et al. Molecular characterization of a 23-kilodalton major antigen secreted by Toxoplasma gondii. Proc Natl Acad Sci USA. 1989;86:7537-41.

28. Beghetto E, Pucci A, Minenkova O, Spadoni A, Bruno L, Buffolano W, et al. Identification of a human immunodominant B-cell epitope within the GRA1 antigen of Toxoplasma gondii by phage display of CDNA libraries. Int J Parasitol. 2001:31:1659-68.

29. Wang Y, Wang G, Ou J, Yin H, Zhang D. Analyzing and identifying novel B cell epitopes within Toxoplasma gondii GRA4. Parasit Vectors. 2014;7:474.

30. Altcheh J, Diaz NS, Pepe CM, Martin V, Nigro M, Freilij H, et al. Kinetic analysis of the humoral immune response against 3 Toxoplasma gondii-recombinant proteins in infants with suspected congenital toxoplasmosis. Diagn Microbiol Infect Dis. 2006;56:161-5.

31. Chardès T, Bourguin I, Mevelec MN, Dubremetz JF, Bout D. Antibody responses to Toxoplasma gondii in sera, intestinal secretions, and milk from orally infected mice and characterization of target antigens. Infect Immun. 1990;58:1240-6.

32. Saavedra R, Becerril MA, Dubeaux C, Lippens R, De Vos MJ, Hérion P, et al. Epitopes recognized by human T lymphocytes in the ROP2 protein antigen of Toxoplasma gondii. Infect Immun. 1996;64:3858-62.

33. Nie H, Fang R, Xiong BQ, Wang LX, Hu M, Zhou YQ, et al. Immunogenicity and protective efficacy of two recombinant pseudorabies viruses expressing Toxoplasma gondii SAG1 and MIC3 proteins. Vet Parasitol. 2011;181:215-21.

34. Jiang T, Gong D, Ma LA, Nie H, Zhou Y, Yao B, et al. Evaluation of a recombinant MIC3 based latex agglutination test for the rapid serodiagnosis of Toxoplasma gondii infection in swines. Vet Parasitol. 2008;158:51-6.

35. Fang $R$, Nie H, Wang Z, Tu P, Zhou D, Wang L, et al. Protective immune response in BALB/C mice induced by a suicidal DNA vaccine of the MIC3 gene of Toxoplasma gondii. Vet Parasitol. 2009;164:134-40.

36. Tenter AM, Heckeroth AR, Weiss LM. Toxoplasma gondii: from animals to humans. Int J Parasitol. 2000;30:1217-58.

37. Pipia AP, Varcasia A, Dessì G, Panzalis R, Gai C, Nonnis F, et al. Seroepidemiological and biomolecular survey on Toxoplasma gondii infection on organic pig farms. Parasitol Res. 2018;117:1637-41.

38. Gazzonis AL, Marangi M, Villa L, Ragona ME, Olivieri E, Zanzani SA, et al. Toxoplasma gondii infection and biosecurity levels in fattening pigs and sows: serological and molecular epidemiology in the intensive pig industry (Lombardy, Northern Italy). Parasitol Res. 2018;117:539-46.

39. Holec-Gasior L. Toxoplasma gondii recombinant antigens as tools for serodiagnosis of human toxoplasmosis: current status of studies. Clin Vaccine Immunol. 2013;20:1343-51.

40. Verhelst D, De Craeye S, Dorny P, Melkebeek V, Goddeeris B, Cox E, et al. IFN- $\gamma$ expression and infectivity of Toxoplasma infected tissues are associated with an antibody response against GRA7 in experimentally infected pigs. Vet Parasitol. 2011:179:14-21.

\section{Publisher's Note}

Springer Nature remains neutral with regard to jurisdictional claims in published maps and institutional affiliations. 